\title{
L'expression des émotions dans les langues hmong : l'exemple des psycho-collocations du hmong blanc et du hmong noir
}

\author{
Léa Mouton ${ }^{1, *}$ \\ ${ }^{1}$ Laboratoire Dynamique Du Langage, Université Lumière Lyon 2, 69007 Lyon, France
}

\begin{abstract}
Résumé. Cet article propose une description des constructions qui dénotent métaphoriquement des émotions en faisant une référence explicite à une partie du corps ou un organe. Quoi que les expressions idiomatiques avec une partie du corps pour parler des émotions soient fréquentes dans les langues du monde, les langues d'Asie du Sud-Est possèdent des constructions spécifiques concernant ce champ lexical où elles suivent un schéma syntaxique récurrent en associant un verbe avec un nom d'organe ou une partie du corps perçu comme le siège des émotions. Ces constructions sont nommées "psycho-collocations » dans les descriptions des langues de cette région. Elles sont également présentes dans les langues hmong. Pour parler des émotions, ces langues (hmong blanc, hmong noir) suivent la tendance générale des autres langues d'Asie du Sud-Est en associant un verbe avec un organe ou une partie du corps en particulier. Néanmoins, elles diffèrent des autres langues d'Asie du SudEst en ce qu'elles possèdent également des termes qui nomment littéralement des types particuliers d'émotions. En effet, les psychocollocations sont dans certaines langues de la région les seuls moyens disponibles pour exprimer les émotions.
\end{abstract}

\begin{abstract}
This article offers a description of constructions that metaphorically denote emotions by making an explicit reference to a body part or an organ. Whereas idiomatic expressions with a body part expressing emotions are frequent in the world languages, Southeast Asian languages have specific constructions concerning this lexical domain. These constructions follow a recurring syntactic pattern by associating a verb and a particular organ or body part treated as the seat of emotions or feelings. They are called "psycho-collocations » in the descriptions of the languages of this region. They are also present in Hmong languages. To speak about emotions, these languages (White Hmong, Black Hmong) follow the general tendency of other Southeast Asian languages by associating a verb and a particular organ or body part term. However, they differ from other Southeast Asian languages in that they also have terms that literally name particular types of emotions. In some languages of this region, psycho-collocations are the only resource available to express emotions.
\end{abstract}

\footnotetext{
*1ea.mouton@yahoo.fr
} 


\section{Introduction: l'expression des émotions et leur relation au corps}

\subsection{Les parties du corps: source métaphorique des émotions dans les langues du monde}

Selon le Trésor de la Langue Française informatisé (TLFi) [1], l'émotion peut être définie comme une «[c] onduite réactive, réflexe, involontaire vécue simultanément au niveau du corps d'une manière plus ou moins violente et affectivement sur le mode du plaisir ou de la douleur ». Selon cette définition, l'émotion met en jeu le corps de l'expérienceur. En effet, les émotions sont généralement accompagnées de symptômes physiques visibles, tel le cœur qui bat plus vite à cause de la peur. Ponsonnet [2, pp. 13-14] définie plus précisément l'émotion comme des états internes, privés et invisibles qui se manifestent par des comportements externes observables. Elle offre une définition des émotions qui inclue des termes renvoyant à des états émotionnels durables (i.e. se sentir mal en s'inquiétant pour quelqu'un, être en deuil), des dispositions émotionnelles (tendance pour un individu à vivre un état émotionnel particulier, i.e. être enclin à la colère) ou encore des mots qui renvoient à des événements ou épisodes émotionnels (i.e. se sentir satisfait pendant un bref moment). Par ailleurs, l'auteur n'émet pas de distinction entre «émotion » et «sentiment ». Ce dernier peut être défini selon le (TLFi) [1], comme un «[é]tat affectif complexe, assez stable et durable, composé d'éléments intellectuels, émotifs ou moraux, et qui concerne le "moi" (orgueil, jalousie...) soit autrui (amour, envie, haine...). »

Il n'est donc pas surprenant que les langues du monde utilisent certaines parties du corps ou certains organes comme source métaphorique dans l'expression des états émotionnels et affectifs. C'est le cas des langues indo-européennes, comme le français qui utilise le foie pour parler de la peur ou l'anglais qui décrit littéralement un cœur déchiré, mais qui renvoie métaphoriquement à un chagrin d'amour.
a. français avoir les foies (blancs) 'avoir peur'
b. anglais heartbreak
'chagrin d'amour'
(lit. déchirement de cœur)

En outre, Wierzbicka [3, p. 36] postule que l'utilisation des parties du corps dans l'expression des émotions est très fréquente dans les langues du monde, voire une caractéristique universelle.

Les langues d'Asie du Sud-Est répondent également à cette caractéristique puisqu'elles présentent, au sein du champ sémantique des émotions ou des sentiments, un ensemble de constructions particulières contenant un terme renvoyant à une partie du corps (voir section suivante).

$\mathrm{Au}$ vu de la littérature, ce thème a été plutôt bien couvert pour les langues d'Asie du Sud-Est [4], [5], [6], [7], [8], [9] et les langues australiennes [10]. Les études sur l'Asie du Sud-Est constituent des études de langues individuelles. Les travaux de Ponsonnet \& Laginha [10] présentent une étude pionnière sur 67 langues d'Australie et proposent une typologie des expressions émotionnelles contenant des parties du corps.

\subsection{Trait aréal des langues d'Asie du Sud-Est}

L'utilisation de constructions contenant une partie du coup pour exprimer les émotions constituent l'un des traits aréaux des langues d'Asie du Sud-Est. D'une part, car elles se rencontrent dans les cinq familles de langues présentes dans cette aire linguistique (sinotibétaine, tai-kadai, hmong-mien, austro-asiatique et austronésienne). D'autre part, car elles 
suivent un schéma syntaxique spécifique et récurrent. Les exemples suivants illustrent ces constructions très répandues dans cette région.

BIRMAN (SINO-TIBETAINE [11])

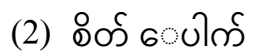

sei? pao?

esprit exploser

'se mettre en colère'

THAI (TAI-KADAI, [12])

(3) š̌ə cay

gâcher cœur

'triste, malheureux 'se'

HMONG LENG (HMONG-MIEN, [13])

(4) chim sab

ê.fâché foie

'être en colère, être

contrarié'

VIETNAMIEN (AUSTRO-ASIATIQUE, [14])

(5) vui lòng

ê.joyeux entrailles

'être heureux'se'

Cham (Austronesienne, [15])

(6) prŏy ko?

large tête

'être $\operatorname{arrogant}(\mathrm{e})$ '

Ces exemples montrent que les langues de cette région associent une partie du corps ou un organe avec un adjectif ou un verbe pour dénoter des émotions ou des sentiments. Ces constructions de schéma syntaxique spécifique sont appelées «psycho-collocations » dans les descriptions des langues d'Asie du Sud-Est [4, p. 9].

De plus, ce phénomène est souvent le seul moyen disponible pour exprimer les émotions. Il semble, en effet, que les langues d'Asie du Sud-Est qui possèdent des psychocollocations aient tendance à préférer leur utilisation par rapport aux lexèmes nommant littéralement des émotions (heureux, triste) lorsqu'elles en possèdent. C'est le cas du birman, qui ne possède que deux verbes pour dire 'aimer' et 'avoir peur, être effrayé' (Vittrant, p.c.).

\subsection{Définition des psycho-collocations}

Le terme «psycho-collocation » a été forgé par Matisoff [4] pour décrire des collocations qui font référence à une émotion (ou état psychologique ${ }^{\dagger}$ ). Les collocations sont définies

$\dagger$ Dans les langues hmong, les psycho-collocations dénotent également des états mentaux (être plein de soucis), des états physiques (avoir le frisson), des état psychologiques (être nostalgique), des propriétés psychologiques (être mauvais) ou encore des traits de caractère 
comme des « cooccurrences privilégiées de deux constituants linguistiques entretenant une relation sémantique et syntaxique » $[16$, p. 33]. En effet, ces constructions polymorphémiques combinent un nom d'organe ou une partie du corps appelé «psychonoun » (qui sera traduit par «psycho-nom ») avec un morphème prédicatif (généralement un adjectif ou un verbe) que Matisoff [4, p. 9] appelle " psycho-mate ». Le terme «psychomate », peu transparent, sera traduit par "psycho-verbe» dans la suite de cet article, puisque le morphème accompagnant le psycho-nom est un verbe (statif ou actif).

$$
\text { psycho-nom }+ \text { psycho-verbe }=\text { psycho-collocation }
$$

Par exemple en lahu (famille des langues tibéto-birmanes, branche lolo-birmane, parlé notamment en Chine), nima lù 'être déprimé' est composé du nom 'cœur' et du verbe 'détruire'.

LAHU [4, p. 10]

(7) ni.ma lù
cœur détruire
'être déprimé(e)'

Les travaux sur les langues d'Asie du Sud-Est montrent que chaque langue semble privilégier un endroit particulier du corps comme étant la source métaphorique des émotions ou des sentiments. Le cœur est privilégié en thaï [4], le foie est préféré en malais [6], l'esprit en birman [9] et les intestins ou l'estomac sont favorisés en vietnamien [7].

\subsection{L'expression des émotions dans les langues hmong}

La famille des langues hmong fait partie de la famille des langues hmong-mien (branche hmongique). Les langues hmong présentent de nombreuses formes dialectales (hmong blanc, hmong vert, hmong leng, hmong noir) très proches les unes des autres avec quelques variations lexicales et phonologiques [17]. Elles sont parlées par plus de dix millions de locuteurs dispersés en Asie du Sud-Est, en Chine, en Thaïlande, au Laos et au Vietnam. On trouve encore des locuteurs en France, en Guyane française, aux États-Unis et en Australie [18]. Le hmong blanc et le hmong vert sont des variétés parlées en Thaïlande, au Laos et par la diaspora occidentale ; des variétés de hmong leng et de hmong noir ont été identifiées au Laos et au Vietnam.

Dans les langues hmong, les émotions peuvent s'exprimer par deux constructions : des lexèmes monomorphémiques ou des psycho-collocations (8).

Par exemple, le hmong noir présente des termes qui nomment littéralement des types particuliers d'émotions comme ntshai 'avoir peur', hlub 'aimer' et mob 'douleur, avoir mal, faire mal'.

Outre ces lexèmes, les langues hmong utilisent les psycho-collocations pour exprimer des émotions. Par exemple en hmong blanc, puv siab 'être satisfait' est composé du verbe statif 'être plein' et du nom 'foie' [5, p. 170].

HMONG BLANC

(8)

$$
\begin{aligned}
& \text { puv siab } \\
& \text { ê.plein foie } \\
& \text { 'être satisfait(e), content(e), heureux·se' } \\
& \text { lit. être plein dans le foie }
\end{aligned}
$$

(personne pure et sans faute), voir [5, p. 161] et [13, p. 618]. Les exemples donnés dans cet article illustreront uniquement des psycho-collocations exprimant des émotions. 
D'un point de vue aréal, la présence de lexèmes monomorphémiques (non compositionnels) nommant littéralement des émotions dans les langues hmong constitue une particularité, car les langues d'Asie du Sud-Est ne possèdent souvent que les psychocollocations concernant ce champ lexical [9, p. 264].

La suite de cet article propose une étude des psycho-collocations des langues hmong, en particulier le hmong blanc et le hmong noir. La partie suivante s'intéresse au fonctionnement syntaxique de ces collocations qui expriment métaphoriquement les émotions. Elle présente les noms et les verbes qui prennent respectivement les places du psycho-nom et du psycho-verbe dans ces constructions. À l'instar des autres langues d'Asie du Sud-Est, quelles parties du corps sont mises en jeu dans les psycho-collocations des langues hmong ? Quel est le locus favori des émotions pour les locuteurs de ces langues? Les psycho-collocations montrent ensuite qu'elles acceptent deux ordres des constituants qui les composent : l'ordre verbe-nom ou l'ordre nom-verbe. Chaque ordre semble être associé à un type d'émotion particulier (émotion de base ou émotion moins prototypique). Enfin, cette partie finit par présenter trois manières différentes de former des émotions antonymes. La dernière partie propose quelques analyses préliminaires centrées sur le hmong noir, une variété de hmong parlée au Vietnam. Pour parler des émotions, cette langue est typique des langues hmong puisqu'elle possède des lexèmes qui nomment littéralement les émotions et présente également un ensemble de psycho-collocations. Dans ces dernières, certaines structures qui dénomment les émotions montrent un sens littéral redondant tandis que d'autres reflètent un sémantisme non transparent. En outre, un aspect qui n'a pas été étudié dans les précédents travaux sur les langues hmong est discuté, comme le processus de nominalisation qui permet de former des noms d'affects à partir des états émotionnels fonctionnant comme des prédicats.

\section{Les psycho-collocations dans les langues hmong}

Les données de hmong présentées dans cette partie proviennent majoritairement du hmong blanc parlé au Laos et par la diaspora occidentale. Elles sont complétées par des données de hmong noir, une variété de hmong parlée au Vietnam qui n'a, à ce jour, fait l'objet d'aucun travail de description linguistique. Pour les données du hmong blanc, deux sources sont utilisées : une étude sur les psycho-collocations en hmong blanc [5] et le dictionnaire hmong blanc-français [19]. Les données de hmong noir sont des données de première main collectées sur le terrain dans la région de Sapa située dans le Nord du Vietnam.

Les langues hmong suivent la tendance générale des langues d'Asie du Sud-Est puisqu'elles possèdent des psycho-collocations pour exprimer les émotions, lesquelles associent une partie du corps ou un organe avec un verbe comme dans l'exemple (9). HMONG BLANC

(9)
zoo siab
ê.bon foie
'être content(e), heureux·se'
lit. être bon(ne) dans le foie

\subsection{Les psycho-noms}

Dans les langues hmong, le foie est clairement l'organe favori mis en jeu dans les psychocollocations. La lecture du dictionnaire hmong blanc-français [19] a révélé 182 psychocollocations contenant l'organe siab 'foie' sur les 215 psycho-collocations listées, soit 
environ $85 \%$. Certaines constructions contenant le nom 'foie' sont employées pour exprimer des émotions de bases dans les langues hmong (10) à (14).

HMONG NOIR

(10) txuj

CLF

kev

zoo

sab

'la joie'

ê.bon foie

'la joie'

(11) tu

séparer foie

'être triste, malheureux · se'

lit. séparer dans le foie

HMONG BLANC

(12) kuv

$1 \mathrm{sg}$

lub

clf:3d

ê.content/ê.heureux

'mon désir profond'

lit. mon foie veut

(13) $1 w j$

ê.pourri

siab

foie

'être contrarié(e), être dégoûté(e)'

$\begin{array}{ll}\text { siab } & \text { xav } \\ \text { foie } & \text { vouloir }\end{array}$

lit. 'être pourri(e) dans le foie et pourri(e) dans le cœur'

(14) chim siab

ê.fâché foie

'être en colère, contrarié( $(e)$ '

lit. être fâché(e) dans le foie

Dans les autres psycho-collocations, faisant référence à des émotions, relevées à partir du dictionnaire hmong blanc-français [19], les parties du corps mobilisées sont des parties du visage comme la joue (15), l'œil (16) et le menton (17).

HMONG BLANC

(15) txhawj
avoir.peur
txhawj plhu
dub
'avoir le visage violet d'appréhension'
ê.noir
lit. avoir peur et (avoir) la joue noire

(16) qhov.muag

oeil

'être gêné(e)'

lit. l'œil est rouge liab

ê.rouge

(17) tsiv

tsaim

ê.méchant

'être colérique'

menton

lit. être méchant(e) dans le menton

Le tableau 1 recense les parties du corps présentes dans les psycho-collocations exprimant des émotions du dictionnaire hmong blanc-français [19]. Il donne leur nombre d'occurrence et le type d'émotion auquel elles sont associées. 


\begin{tabular}{|c|c|c|}
\hline Organe ou partie du corps & Nombre d'occurrences & Émotion associée \\
\hline Foie & $182(84,7 \%)$ & $\begin{array}{l}\text { - joie } \\
\text { - tristesse } \\
\text { - peur } \\
\text { - colère } \\
\text { - dégoût } \\
\text { - surprise } \\
\text { - inquiétude } \\
\text { - désir }\end{array}$ \\
\hline Joue & $10(4,6 \%)$ & $\begin{array}{l}\text { - peur } \\
\text { - colère }\end{array}$ \\
\hline Eil & $5(2,3 \%)$ & $\begin{array}{l}\text { - tristesse } \\
\text { - jalousie } \\
\text { - envie } \\
\text { - honte } \\
\text { - gêne }\end{array}$ \\
\hline Menton & $1(0,5 \%)$ & - colère \\
\hline
\end{tabular}

Tableau 1 : Les organes impliqués dans les psycho-collocations du hmong blanc

Ce tableau montre qu'en hmong blanc, le foie est clairement le siège des émotions. Non seulement par le nombre conséquent de psycho-collocations dans lesquelles il apparait, mais aussi par les types variés d'émotions qui y font une référence explicite. En termes de nombre de constructions, la deuxième partie du corps la plus fréquemment utilisée, pourtant largement moins utilisée que le foie, est la joue. Sémantiquement, elle est utilisée pour deux émotions de base négatives (peur, colère). L'œil est notamment utilisé dans l'expression de la honte et la gêne. Ponsonnet \& Laginha [10] notent que dans la majorité des langues du monde ainsi que dans de nombreuses langues australiennes, la honte a une représentation métaphorique significative dans l'œil. Il n'est donc pas surprenant de retrouver ce type de collocations dans les langues hmong. Enfin il est intéressant de remarquer que la colère est exprimée à travers trois parties du corps : le foie, la joue et le menton.

\subsection{Les psycho-verbes}

Concernant les psycho-verbes, ce sont des verbes statifs qui correspondent à un état comme 'être ébréché' en (18), des états émotionnels comme 'être en colère' en (19) ou une couleur comme 'être noir' en (20) [5, p. 173], [7, p. 551], [19].

HMONG BLANC

$\begin{array}{ll}\text { siab } & \text { khis } \\ \text { foie } & \text { ê.ébréché }\end{array}$

'être peiné(e), attristé(e)'

lit. le foie est ébréché

$\begin{array}{ll}\text { npau } & \text { siab } \\ \text { ê.en.colère } & \text { foie }\end{array}$

'bouillir de colère'

lit. être en colère dans le foie

$\begin{array}{ll}\text { dub } & \text { muag } \\ \text { ê.noir } & \text { œil } \\ \text { 'être triste' } & \end{array}$


lit. être noir(e) dans l'œil

Les psycho-verbes peuvent également être des verbes actifs comme 'tomber' (21) et 'percer' (22) en hmong blanc [5, p. 171], [7, p. 551].

HMONG BLANC

poob siab
tomber foie
'être effrayé(e)'

lit. tomber dans le foie

chob siab
percer foie
'être chagriné(e)'
lit. percer dans le foie

\subsection{Sémantique et ordre des constituants}

Les psycho-collocations des langues hmong peuvent suivre deux ordres : soit l'ordre verbenom, soit l'ordre inverse nom-verbe. Ces constructions montrent un ordre des constituants plutôt rigide associé à un sémantisme particulier. Dans le premier cas, il semble que l'ordre verbe-nom corresponde à des émotions basiques comme la joie, la tristesse, la colère ou la peur. Dans l'autre, les constructions nom-verbes semblent exprimer des émotions moins prototypiques comme le désir, la gêne, l'appréhension (voir l'annexe 2 qui donne la liste des psycho-collocations du hmong blanc utilisées dans cet article). Toutefois, cette hypothèse nécessiterait d'être vérifiée par un examen complet de l'ensemble des psychocollocations des langues hmong.

Néanmoins, il existe dans ces langues des paires de psycho-collocations où psycho-nom et psycho-verbe sont inversés. L'émotion qui résulte de ces collocations est différente comme le montrent les exemples (23) du hmong blanc ; poob siab signifie 'être effrayé' (a), et siab poob signifie 'être découragé, affligé' (b) [5, p. 177].

HMONG BLANC

\begin{tabular}{lll} 
(23) a. & poob & \multicolumn{1}{c}{ siab } \\
& tomber & foie \\
& 'être effrayé(e)' \\
& lit. tomber dans le foie \\
b. & siab & poob \\
& foie & tomber \\
& 'être découragé(e), affligé(e)' \\
& lit. le foie tombe
\end{tabular}

D'autres paires de psycho-collocations où psycho-nom et psycho-verbe sont inversés évoquent des synonymes d'une même émotion comme la tristesse, la peine (24) [5, p. 173], [7, p. 557].

HMONG BLANC

$$
\begin{array}{ll}
\text { siab } & \text { khis } \\
\text { foie } & \text { ê.ébréché } \\
\text { 'être peiné(e), attristé(e)' }
\end{array}
$$


lit. le foie est ébréché

b. khis siab

ê.ébréché foie

'être peiné(e), affligé(e)'

lit. être ébréché(e) dans le foie

Par ailleurs, il existe des constructions où l'inversion du psycho-nom avec le psychoverbe ne provoque aucun changement de sens, tawg siab 'être cassé + foie' ou siab tawg 'foie + être cassé' signifient indifféremment 'être étonné' [19].

\subsection{Formation d'antonymes}

Dans les langues hmong, les états émotionnels antonymes peuvent être formés grâce à des psycho-verbes antonymes. Dans les exemples (25) du hmong blanc, le psycho-verbe $x u$ 'manquer sa cible' en association avec le foie signifie 'être mécontent, malheureux'(a) et son antonyme raug 'atteindre sa cible' en association avec le foie signifie 'être content, heureux' (b) [5, pp. 170-170].

HMONG BLANC
a. $\mathbf{x u}$
siab
manquer sa.cible
foie
'être mécontent(e), malheureux·se'
lit. manquer sa cible dans le foie
b. raug siab
atteindre.sa.cible foie
'être content (e), heureux·se'
lit. atteindre sa cible dans le foie

Les émotions antonymes peuvent également être formées par l'ajout du marqueur de négation tsi devant la psycho-collocation comme dans l'exemple (26b) du hmong noir.

HMONG NOIR
a. nyaab.laaj yeej
vietnam
zoo
ê.bon
sab
kaws li

\section{ê.content/ê.heureux}

'Les Vietnamiens sont très heureux'
b. kuv
tsi
zoo
sab
$1 \mathrm{SG}$
NEG
ê.bon
foie

\section{ê.content/ê.heureux}

'Je suis malheureuse'

lit. Je ne suis pas heureuse

Une troisième manière de créer des antonymes est l'utilisation de psycho-verbes différents comme dans les exemples (27). En hmong noir le prédicat 'être bon' en association avec le foie signifie 'être heureux' (a) et le verbe 'séparer' en association avec le même nom d'organe 'foie' signifie 'être malheureux'(b).

HMONG NOIR

$\begin{array}{lll}\text { (27) a. } & \text { zoo } & \text { sab } \\ & \text { ê.bon } & \text { foie }\end{array}$ 
'être content(e),

heureux. se'

lit. être bon(ne) dans le

foie

$\begin{array}{ll}\text { b. } & \text { tu } \\ \text { séparer fab } \\ \text { 'être triste, } \\ \text { malheureux·se' } \\ \text { lit. séparer dans le foie }\end{array}$

\section{L'expression des émotions en hmong noir}

Cette dernière partie propose quelques analyses préliminaires sur l'expression des émotions en hmong noir. Les données sur lesquelles reposent ces analyses ont été collectées auprès de quatre locutrices du hmong noir vivant dans la région de Sapa située au Nord du Vietnam. Quatre corpus ont été utilisés : une liste de vocabulaire, deux textes oraux (récit personnel, chanson) et une narration collectée à partir d'une histoire illustrée sans texte. Au total, 16 lexèmes (monomorphémiques et psycho-collocations) faisant référence à un état émotionnel ont été identifiés dans cette langue. Une liste exhaustive des lexèmes sera donnée en annexe 1 .

\subsection{Présence de lexèmes monomorphémiques}

Le hmong noir présente un ensemble de lexèmes monomorphémiques exprimant des émotions. Cinq formes ont été identifiées jusqu'à présent. Ces lexèmes peuvent fonctionner comme des noms ou comme des verbes suivant le contexte. Dans les exemples (28), la forme $m o b$ peut signifier 'douleur' (a) ou 'avoir mal, faire mal' (b) suivant le contexte.
a. rov
nco
se.souvenir
qub
mob
iter
ancien
douleur

'(Je) me remémore d'anciennes douleurs'

Lit. (Je) me re-souviens d'anciennes douleurs

b. ze.zog mob yus

tous avoir.mal/faire.mal $1 \mathrm{sg}$

'Tout le monde me fait mal'

Lit. Tous font mal à moi

\subsection{Les psycho-collocations}

Dans cette langue, les émotions peuvent également s'exprimer par des psycho-collocations, tout comme dans les autres langues hmong présentées dans la partie précédente.

Dans les données du hmong noir, les organes ou les parties du corps mis en jeu dans les psycho-collocations sont le foie (4 constructions (29)) et les yeux (1 construction (30)). Quant aux psycho-verbes, ce sont des verbes statifs (a-c) ou des verbes actifs (d). Les verbes statifs expriment des états (être pourri) ou des états émotionnels (être fâché). 


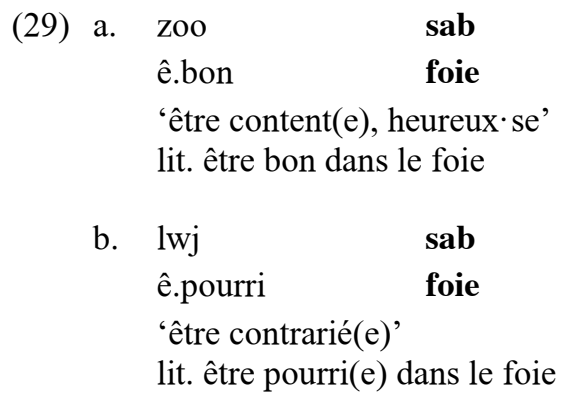

c. chim sab

'être contrarié(e)'

lit. être fâché(e) dans le foie

$\begin{array}{ll}\text { d. } & \text { sub } \\ \text { séparer } & \text { foie }\end{array}$

'être triste, malheureux·se'

lit. séparer dans le foie

De plus, on retrouve soit des constructions dans lesquelles le psycho-verbe a son sens littéral redondant, soit des constructions où l'association du psycho-verbe avec le psychonom n'est sémantiquement pas transparente. En (30) la signification de la collocation a la même signification que le psycho-verbe : nxtaaj muag 'avoir honte' composé de 'avoir honte + œil' signifie littéralement 'avoir honte dans l'œil'. L'exemple (31), quant à lui, montre que la signification de la collocation n'est pas transparente : zoo sab 'être bon + foie' signifie 'être heureux' littéralement 'être bon dans le foie'.
kuv ntxaaj
muag
1SG avoir.honte œil
'J'ai honte'
lit. J'ai honte dans l'œil

(31) 


\subsection{Dérivation nominale autour des émotions}

Les psycho-collocations expriment de base des états émotionnels et affectifs et fonctionnent comme des prédicats. En hmong noir, ces prédicats peuvent être nominalisés. L'ajout de la forme kev 'chemin' sur le prédicat permet ainsi de former des noms d'affects. En (32) le nominalisateur $k e v$ est ajouté aux collocations $l w j s a b$ 'être contrarié' (a), zoo sab 'être content' (b) et $t u s a b$ 'être malheureux' (c) pour former respectivement les noms d'affects 'contrariété', 'joie' et 'peine'. En outre, la forme kev peut également être ajoutée aux lexèmes monomorphémiques comme hlub 'aimer' pour former le nom 'amour' (33).

$$
\begin{array}{llll}
\text { a. } & \text { ib } & \text { txuj } & \text { kev-[lwj-sab] } \\
\text { un } & \text { CLF } & \text { chemin-[ê.pourri-foie] } \\
& & \text { NMLZ-ê.contrarié }
\end{array}
$$

'une contrariété'

b. txuj kev-[zoo-sab]

CLF chemin-[ê.bon-foie]

NMLZ-ê.heureux

'la joie'

c. txuj kev-[tu-sab]

CLF chemin-[séparer-foie]

NMLZ-ê.malheureux

'la peine'

$$
\begin{array}{lll}
\text { ib } & \text { txuj } & \text { kev-hlub } \\
\text { un } & \text { CLF } & \text { chemin-aimer }
\end{array}
$$

'un amour'

\subsection{Forme sab 'foie' seule : 'être triste' ?}

Pour finir, les données du hmong noir montrent une construction particulière pour exprimer la tristesse : l'utilisation de la forme $s a b$ 'foie' seule.

L'exemple ci-dessous rappelle la construction complète pour exprimer la tristesse dans cette langue.
(34) tu
$\mathrm{sab}$
séparer
foie
'être triste,
malheureux'se'
lit. séparer dans le foie

Les locuteurs du hmong noir peuvent aussi utiliser uniquement la forme $s a b$ 'foie' pour exprimer cette émotion. L'exemple (38) signifie 'je suis triste et je pleure', littéralement 'je suis foie et les larmes me viennent'.

(35) kuv yog sab kua.muag los 
1SG être foie larme venir

'Je suis triste et je pleure'

lit. Je suis foie et (les) larmes (me) viennent

Par ailleurs, la réduplication (processus courant et très productif dans les langues hmong en général) de la forme $s a b$ 'foie' en (39) provoque une intensification de l'état émotionnel.

nyuas dev sab sab
petit chien foie REDUP
'Le petit chien est très triste'
lit. (Le) petit chien (est) très foie

Ces deux exemples montrent que la forme $s a b$, lorsqu'elle apparait seule, pourrait avoir par défaut la signification de base 'être triste'. Toutefois, le corpus du hmong noir contient seulement ces deux occurrences où cette forme apparaît seule. Cette hypothèse mériterait une analyse approfondie à la lumière de nouvelles données.

\section{Conclusion}

Pour commencer, cet article a présenté les psycho-collocations qui sont des constructions typiques des langues d'Asie du Sud-Est présentes dans les cinq familles de langues de cette région. Elles représentent, d'ailleurs dans certaines langues, les seuls moyens disponibles pour exprimer les émotions. Ces constructions font référence à un état émotionnel ou affectif et utilisent une partie du corps ou un organe particulier comme siège des émotions.

Les émotions représentent un domaine lexical important dans les langues hmong présentées dans cette étude (hmong blanc, hmong noir). Pour parler des émotions, ces langues utilisent fréquemment les psycho-collocations qui associent un organe ou une partie du corps avec un verbe actif ou statif, mais aussi des lexèmes monomorphémiques de sémantisme abstrait. L'usage de ces derniers constitue un trait particulier dans l'expression des émotions pour les langues d'Asie du Sud-Est, car la majorité de ces langues possèdent uniquement les psycho-collocations.

$\mathrm{Au}$ sein des psycho-collocations des langues hmong qui expriment des émotions, 4 organes ou parties du corps ont été identifiés toutes langues confondues : le foie, la joue, l'œil et le menton. Le foie est clairement l'organe préféré puisqu'il a été relevé dans plus de $80 \%$ des constructions du hmong blanc. Dans une moindre mesure, les deuxième et troisième parties du corps les plus fréquemment utilisées sont la joue, et l'œil. L'œil est utilisé pour exprimer métaphoriquement la honte ou la gêne, une caractéristique qui se retrouve dans la plupart des langues du monde. Quant au menton, il a été retrouvé dans une psycho-collocation pour exprimer la colère.

En résumé, cet article a montré que le hmong noir est typique des langues hmong puisqu'il possède des psycho-collocations (voir l'annexe 1 qui donne une liste exhaustive des psycho-collocations du hmong noir identifiées jusqu'à présent). Il a également évoqué le processus de nominalisation par l'ajout de la forme kev 'chemin' sur le prédicat exprimant un état émotionnel. Cette caractéristique n'a pas été relevée dans les travaux existants sur le hmong blanc. Enfin, il semblerait que les locuteurs du hmong noir utilisent la forme $s a b$ 'foie' seule pour exprimer l'émotion de tristesse.

\section{Abréviations}

$1 \quad 1^{\text {re }}$ personne

$2 \quad 2^{\mathrm{e}}$ personne

CLF classificateur 


$\begin{array}{ll}\text { IRR } & \text { irréel } \\ \text { ITER } & \text { aspect itératif } \\ \mathrm{N} & \text { nom } \\ \mathrm{NMLZ} & \text { nominalisateur } \\ \mathrm{NUM} & \text { numéral } \\ \mathrm{NEG} & \text { négation } \\ \mathrm{O} & \text { objet } \\ \text { REDUP } & \text { réduplication } \\ \mathrm{S} & \text { sujet } \\ \mathrm{SG} & \text { singulier } \\ \mathrm{V} & \text { verbe }\end{array}$

\section{Bibliographie}

1. TLFi, Définition d' "émotion », Dans Trésor de La Langue Française informatisé (TLFi), ATILF - CNRS \& Université de Lorraine. URL : http://stella.atilf.fr/Dendien/scripts/tlfiv5/advanced.exe?8;s=3392510565;

2. M. Ponsonnet, The language of emotions in Dalabon (Northern Australia), Thèse de Doctorat, The Australian National University (Canberra, 2013).

3. A. Wierzbicka, Emotion across languages and cultures: diversity and universals, Cambridge, (Cambridge University Press, Cambridge, 1999).

4. J. A. Matisoff, Hearts and minds in South-East Asian languages and english: an essay in the comparative lexical semantics of psycho-collocations, CLAO, 15(1), 5-57, (1986).

5. A. Jaisser, DeLIVERing an introduction to psycho-collocations with SIAB in Hmong, LTBA, 13 (1), 159-178 (1990).

6. E. Oey, Psycho-collocations in Malay, LTBA, 13 (1), 141-158 (1990).

7. M. Clark, «Where do you feel? Stative verbs and body-parts terms in Mainland Southeast Asia », dans H. Chappell \& W. McGregor (éds.), The Grammar of Inalienability, A Typological Perspective on Body Part Terms and the Part-Whole Relation, 529-564, (De Gruyter Mouton, Berlin, 1996).

8. K. Van-Bik, Lai psycho-collocation, LTBA, 21 (1), 201-233 (1998).

9. A. Vittrant, Psycho-collocational expressives in Burmese, dans J. P. Williams (éd.), The Aesthetics of Grammar: Sound and Meaning in the Languages of Mainland Southeast Asia, 255-279, (Cambridge University Press, Cambridge, 2014).

10. M. Ponsonnet \& K. Laginha, The role of the body in descriptions of emotions. A typology of the Australian continent, PC, (accepté).

11. A. Vittrant, Burmese, dans A. Vittrant \& J. Watkins (éds.), The Mainland Southeast Asia Linguistic Area, 56-131, (De Gruyter Mouton, Berlin, 2019)

12. M. Jenny, Thai, dans A. Vittrant \& J. Watkins (éds.), The Mainland Southeast Asia Linguistic Area, 559-608 (De Gruyter Mouton, Berlin, 2019)

13. D. Mortensen, Hmong (Mong Leng), dans A. Vittrant \& J. Watkins (éds.), The Mainland Southeast Asia Linguistic Area, 609-652, (De Gruyter Mouton, Berlin, 2019)

14. D. T. Do-Hurinville \& H. L. Dao, Vietnamese, dans A. Vittrant \& J. Watkins (éds.), The Mainland Southeast Asia Linguistic Area, 384-431, (De Gruyter Mouton, Berlin, 2019). 
15. M. Brunelle \& Phú Văn Hẳn, Colloquial Eastern Cham, dans A. Vittrant \& J. Watkins (éds.), The Mainland Southeast Asia Linguistic Area, 523-558, (De Gruyter Mouton, Berlin, 2019).

16. A. Tutin, I. Novakova, F. Grossmann \& C. Cavalla, Esquisse de typologie des noms d'affect à partir de leurs propriétés combinatoires, LF, 150, 32-49, (2006).

17. B. Niederer, « La langue hmong », A, 26/27, 345-381, (2001-2002).

18. J. Lemoine, «What is the actual number of the $(\mathrm{H})$ mong in the Word », HSJ, 6, 1-8, (2005).

19. Y. Bertrais, Dictionnaire Hmong-Français, (Imprimerie de l'Assomption, Bangkok, 1964). 


\section{Annexe 1 : liste des émotions en hmong noir}

\section{Lexèmes monomorphémiques}

$\underline{\text { Noms }}$

mob

'douleur'

Pouvant fonctionner comme psycho-verbe

$\begin{array}{ll}\frac{\text { Verbes }}{\text { mob }} & \begin{array}{l}\text { 'avoir mal, faire } \\ \text { mal' }\end{array} \\ \text { hlub } & \text { 'aimer' } \\ \text { ntshai } & \text { 'avoir peur' }\end{array}$

ntxaaj 'avoir honte'

\section{Psycho-collocations avec sab 'foie'}

- Psycho-verbe : verbe statif exprimant un état

\begin{tabular}{|c|c|c|}
\hline $\begin{array}{l}\text { lwj } \\
\text { être pourri }\end{array}$ & $\begin{array}{l}\text { sab } \\
\text { foie }\end{array}$ & 'être contrarié' \\
\hline zoo & sab & 'être content, heureux' \\
\hline
\end{tabular}

- Psycho-verbe : verbe statif exprimant une émotion

\begin{tabular}{lll}
\hline chim & sab & 'être contrarié' \\
être fâché & foie &
\end{tabular}

- Psycho-verbe : verbe actif

\begin{tabular}{|c|c|c|c|}
\hline $\begin{array}{l}\text { tu } \\
\text { séparer }\end{array}$ & $\begin{array}{l}\text { sab } \\
\text { foie }\end{array}$ & & 'être triste, malheureux' \\
\hline sab & 'foie' & 1 & 'être triste' \\
\hline
\end{tabular}

Psycho-collocation avec muag 'œil'

$\begin{array}{lll}\text { ntxaaj } & \text { muag } & \text { 'avoir honte' } \\ \text { avoir honte } & \text { 'al }\end{array}$

Dérivation nominale autour des émotions

- Dérivation des émotions monomorphémiques

\begin{tabular}{|c|c|c|}
\hline $\begin{array}{l}\text { kev } \\
\text { chemin }\end{array}$ & $\begin{array}{l}\text { hlub } \\
\text { aimer }\end{array}$ & 'amour' \\
\hline \multicolumn{3}{|c|}{ - Dérivation des psycho-collocations } \\
\hline $\begin{array}{l}\text { kev } \\
\text { chemin }\end{array}$ & $\begin{array}{l}\text { lwj.sab } \\
\text { être contrarié }\end{array}$ & 'contrariété' \\
\hline $\begin{array}{l}\text { kev } \\
\text { chemin }\end{array}$ & $\begin{array}{l}\text { tu.sab } \\
\text { être triste, malheureux }\end{array}$ & 'peine' \\
\hline $\begin{array}{l}\text { kev } \\
\text { chemin }\end{array}$ & $\begin{array}{l}\text { zoo.sab } \\
\text { être content, heureux }\end{array}$ & 'joie’ \\
\hline
\end{tabular}




\section{Annexe 2: liste non exhaustive des psycho-collocations du hmong blanc}

\begin{tabular}{|c|c|c|}
\hline \multicolumn{3}{|c|}{ Psycho-verbe + Psycho-nom } \\
\hline \multicolumn{3}{|l|}{ Chagrin } \\
\hline $\begin{array}{l}\text { chob } \\
\text { percer }\end{array}$ & $\begin{array}{l}\text { siab } \\
\text { foie }\end{array}$ & $\begin{array}{l}\text { 'être } \\
\text { chagriné' }\end{array}$ \\
\hline \multicolumn{3}{|l|}{ Joie } \\
\hline $\begin{array}{l}\text { zoo } \\
\text { être bon }\end{array}$ & $\begin{array}{l}\text { siab } \\
\text { foie }\end{array}$ & $\begin{array}{l}\text { 'être content, } \\
\text { heureux' }\end{array}$ \\
\hline $\begin{array}{l}\text { raug } \\
\text { atteindre sa } \\
\text { cible }\end{array}$ & $\begin{array}{l}\text { siab } \\
\text { foie }\end{array}$ & $\begin{array}{l}\text { 'être content, } \\
\text { heureux' }\end{array}$ \\
\hline \multicolumn{3}{|l|}{ Tristesse } \\
\hline $\begin{array}{l}\mathrm{xu} \\
\text { manquer sa } \\
\text { cible }\end{array}$ & $\begin{array}{l}\text { siab } \\
\text { foie }\end{array}$ & $\begin{array}{l}\text { 'être } \\
\text { mécontent, } \\
\text { malheureux' }\end{array}$ \\
\hline $\begin{array}{l}\text { dub } \\
\text { être noir }\end{array}$ & $\begin{array}{l}\text { muag } \\
\text { œil }\end{array}$ & 'être triste' \\
\hline
\end{tabular}

\begin{tabular}{lll}
\hline Gêne & & \\
\hline $\begin{array}{l}\text { qhov.muag } \\
\text { œil }\end{array}$ & $\begin{array}{l}\text { liab } \\
\text { être rouge }\end{array}$ & 'être gêné' \\
\hline Appréhension & & \\
\hline plhu & dub & $\begin{array}{l}\text { 'avoir le visage } \\
\text { joue }\end{array}$ \\
& être noir & $\begin{array}{l}\text { violet } \\
\text { d'appréhension' }\end{array}$
\end{tabular}

\begin{tabular}{|c|c|c|}
\hline Colère & & \\
\hline $\begin{array}{l}\text { chim } \\
\text { être fâché }\end{array}$ & $\begin{array}{l}\text { siab } \\
\text { foie }\end{array}$ & $\begin{array}{l}\text { 'être en } \\
\text { colère, } \\
\text { contrarié' }\end{array}$ \\
\hline $\begin{array}{l}\text { npau } \\
\text { être en colère }\end{array}$ & $\begin{array}{l}\text { siab } \\
\text { foie }\end{array}$ & $\begin{array}{l}\text { 'bouillir de } \\
\text { colère' }\end{array}$ \\
\hline $\begin{array}{l}\text { tsiv } \\
\text { être méchant }\end{array}$ & $\begin{array}{l}\text { tsaim } \\
\text { menton }\end{array}$ & $\begin{array}{l}\text { 'être } \\
\text { colérique' }\end{array}$ \\
\hline
\end{tabular}




\begin{tabular}{|c|c|c|c|c|}
\hline Étonnement & & & & \\
\hline $\begin{array}{l}\text { tawg } \\
\text { être cassé }\end{array}$ & $\begin{array}{l}\text { siab 'être étonné' } \\
\text { foie }\end{array}$ & $\begin{array}{l}\text { siab } \\
\text { foie }\end{array}$ & $\begin{array}{l}\text { tawg } \\
\text { être cassé }\end{array}$ & 'être étonné' \\
\hline
\end{tabular}

\begin{tabular}{|c|c|c|c|c|}
\hline Peine & & & & \\
\hline $\begin{array}{l}\text { khis } \\
\text { être ébréché }\end{array}$ & $\begin{array}{l}\text { 'être peiné, } \\
\text { affligé' }\end{array}$ & $\begin{array}{l}\text { siab } \\
\text { foie }\end{array}$ & $\begin{array}{l}\text { khis } \\
\text { être ébréché }\end{array}$ & $\begin{array}{l}\text { 'être peiné, } \\
\text { attristé' }\end{array}$ \\
\hline
\end{tabular}

\begin{tabular}{|c|c|c|c|c|c|}
\hline Peur & \multicolumn{5}{|c|}{ Profonde tristesse } \\
\hline $\begin{array}{l}\text { poob } \\
\text { tomber }\end{array}$ & $\begin{array}{l}\text { siab } \\
\text { foie }\end{array}$ & 'être effrayé' & $\begin{array}{l}\text { siab } \\
\text { foie }\end{array}$ & $\begin{array}{l}\text { poob } \\
\text { tomber }\end{array}$ & $\begin{array}{l}\text { 'être découragé } \\
\text { affligé' }\end{array}$ \\
\hline
\end{tabular}

